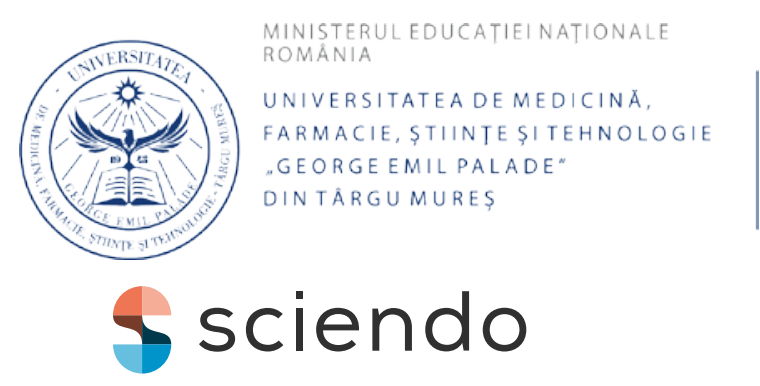

\author{
Acta Marisiensis. SeriaTechnologica \\ Vol. 17 (XXXIV) no. 2, 2020 \\ ISSN 2668-4217, ISSN-L 2668-4217
}

10.2478/amset-2020-0015

\title{
A NOTE ON GENERALIZED SOLUTION OF A CAUCHY PROBLEM GIVEN BY A NONHOMOGENEOUS LINEAR DIFFERENTIAL SYSTEM
}

\author{
Marcel BOGDAN \\ University of Medicine, Pharmacy, Science and Technology "G.E. Palade" of Tîrgu Mureş \\ Nicolae Iorga Street, no. 1, 540088, Mures county, Romania \\ marcel.bogdan@umfst.ro
}

\begin{abstract}
Generalized solution of a Cauchy problem given by a nonhomogeneous linear differential system is recovered to this approach. It considers the case of the free term having at most countable number of discontinuity points. The method, called successive approach, uses the solution on the previous interval (except the first one) for the condition on the given interval. The sequence of commands for a computer algebra system to this method is given.
\end{abstract}

Keywords: Cauchy problem, generalized solution, differential system, Laplace transform, computer algebra system.

\section{The notion of solution}

In the present note, a nonhomogeneous linear differential system is considered. Many handbooks contain methods for solving the homogeneous linear differential system and the Cauchy problem attached, along with the nonhomogeneous case right on. Except that, not always the situation when the nonhomogeneous term is discontinuous is included (one can consult $[3,8,9,10,11])$. And, when it does, the discontinuities are expressed using the Heaviside function [4, 13]. Along with the two known methods, Lagrange's method and Laplace transform, another method, called successive approach, is presented.
Some previous works are referred to our purpose $[1,2]$.

Let $m \in \mathbb{N}=\{1,2, \ldots\}, A=\left(a_{i j}\right)_{1 \leq i, j \leq m} \in$ $M_{m}(\mathbb{R})$, let $I \subseteq[0,+\infty)$ be a nonempty interval with $0 \in I$, let $f_{i}: I \rightarrow \mathbb{R}, 1 \leq i \leq m$, be piecewise continuous functions on $I$, i.e. continuous except at most countable number of (discontinuity) points, and let $\tilde{y}_{i} \in \mathbb{R}, 1 \leq i \leq m$ be given. Let us consider the Cauchy problem $(P C O D S)$

$$
\left\{\begin{array}{l}
y_{1}^{\prime}=a_{11} \cdot y_{1}+a_{12} \cdot y_{2}+\cdots+a_{1 m} \cdot y_{m}+f_{1} \\
y_{2}^{\prime}=a_{21} \cdot y_{1}+a_{22} \cdot y_{2}+\cdots+a_{2 m} \cdot y_{m}+f_{2} \\
\cdots \\
y_{m}^{\prime}=a_{m 1} \cdot y_{1}+a_{m 2} \cdot y_{2}+\cdots+a_{m m} \cdot y_{m}+f_{m} \\
y_{i}(0)=\tilde{y}_{i}, \quad 1 \leq i \leq m .
\end{array}\right.
$$


Let $N \subseteq \mathbb{N}$ and let $\left\{I_{0}, I_{k}\right\}_{k \in N}$ be the partition of $I$, so $I=I_{0} \bigcup\left(\cup_{k \in N} I_{k}\right)$, of the form $I_{0}=\left[0, x_{1}\right)$, $I_{k}=\left[x_{k}, x_{k+1}\right)$, be such that $\left.f_{i}\right|_{I_{k}}$ is continuous $\forall i \in$ $\{1, \ldots, m\}, \forall k \in N$.

Definition 1. A function $Y=\left(y_{1}, \ldots, y_{m}\right)^{T}: I \rightarrow$ $\mathbb{R}^{m}$ is said to be generalized classic solution for $(P C O D S)$ if all the following three conditions are verified:

1. $Y \in C^{1}\left(I_{k}, \mathbb{R}^{m}\right), \forall k \in N \cup\{0\}$ and $Y \in$ $C\left(I, \mathbb{R}^{m}\right)$

2. $Y(0)=\left(\tilde{y}_{1}, \ldots, \tilde{y}_{m}\right)^{T}$;

3. $Y^{\prime}(x)=A \cdot Y(x)+\left[f_{1}(x), \ldots, f_{m}(x)\right]^{T}, \forall x \in$ $I \backslash\left\{x_{k}\right\}_{k \in N}$.

Note that, if $N=\emptyset$, then the notion above reduces to the $C^{1}$ classic solution. Denote by $\tilde{Y}_{0}=$ $\left(\tilde{y}_{1}, \ldots, \tilde{y}_{m}\right)^{T}$ and $f=\left(f_{1}, \ldots, f_{m}\right)^{T}$, so $(P C O D S)$ is written

$$
(P C)\left\{\begin{array}{l}
Y^{\prime}=A \cdot Y+f \\
Y(0)=\tilde{Y}_{0}
\end{array}\right.
$$

In the next section the possibilities of solving $(P C O D S)$ are given, among them the successive approach. In section 3, some examples are used to running the method. In section 4 the input lines are given to be executed by the computer algebra system WolframAlpha, in order to obtain the displayed solutions on the intervals. The next section aims to point out the alternative and the gap to distributional solutions. The last section concludes the idea of the article.

\section{Successive approach}

In the present note, the possibilities of solving $(P C O D S)$ are expressed: I) Formally, by using Laplace transform; II) By Lagrange's method; III) By solving successive Cauchy problems.

Observe that, the substitution method $(m \geq 2)$ works, as long as the (possible existing, for $N \neq \emptyset$ ) angular points, are managed carefully. In parallel, the third method is proposed.

I. For the first method, by applying Laplace transform, denoted by $\mathcal{L}$, to each equation of the system, $(P C O D S)$ becomes $(A L S)$, with notation $\mathcal{Y}_{i}=$

$$
\begin{aligned}
& \mathcal{L}\left(y_{i}\right) \\
& \left\{\begin{array}{l}
p \cdot \mathcal{Y}_{1}(p)-y_{1}(0) \\
=a_{11} \cdot \mathcal{Y}_{1}(p)+a_{12} \cdot \mathcal{Y}_{2}(p)+\cdots+a_{1 m} \cdot \mathcal{Y}_{m}(p) \\
+\mathcal{L}\left(f_{1}\right)(p) \\
p \cdot \mathcal{Y}_{2}(p)-y_{2}(0) \\
=a_{21} \cdot \mathcal{Y}_{1}(p)+a_{22} \cdot \mathcal{Y}_{2}(p)+\cdots+a_{2 m} \cdot \mathcal{Y}_{m}(p) \\
+\mathcal{L}\left(f_{2}\right)(p) \\
\cdots \\
p \cdot \mathcal{Y}_{m}(p)-y_{m}(0) \\
=a_{m 1} \cdot \mathcal{Y}_{1}(p)+a_{m 2} \cdot \mathcal{Y}_{2}(p)+\cdots+a_{m m} \cdot \mathcal{Y}_{m}(p) \\
+\mathcal{L}\left(f_{m}\right)(p)
\end{array}\right.
\end{aligned}
$$

The algebraic linear system $(A L S)$ can be written as

$$
\left\{\begin{array}{l}
\left(p-a_{11}\right) \cdot \mathcal{Y}_{1}(p)-a_{12} \cdot \mathcal{Y}_{2}(p) \\
-\cdots-a_{1 m} \cdot \mathcal{Y}_{m}(p)=\tilde{y}_{1}+\mathcal{L}\left(f_{1}\right)(p) \\
-a_{21} \cdot \mathcal{Y}_{1}(p)+\left(p-a_{22}\right) \cdot \mathcal{Y}_{2}(p) \\
-\cdots-a_{2 m} \cdot \mathcal{Y}_{m}(p)=\tilde{y}_{2}+\mathcal{L}\left(f_{2}\right)(p) \\
\cdots \\
-a_{m 1} \cdot \mathcal{Y}_{1}(p)-a_{m 2} \cdot \mathcal{Y}_{2}(p) \\
-\cdots+\left(p-a_{m m}\right) \cdot \mathcal{Y}_{m}(p)=\tilde{y}_{m}+\mathcal{L}\left(f_{m}\right)(p) .
\end{array}\right.
$$

Its solution is given by the components

$$
\mathcal{Y}_{i}(p)=\frac{1}{\Delta(p)} \cdot \Delta_{i}(p), 1 \leq i \leq m,
$$

where $\Delta(p)=\operatorname{det}\left(p \cdot I_{m}-A\right)=(-1)^{m} \cdot \operatorname{det}(A-p$. $\left.I_{m}\right)$ and $\Delta_{i}(p)=\operatorname{det}\left(\left(p \cdot I_{m}-A\right)_{i} ;\left(\tilde{y}_{i}+\mathcal{L}\left(f_{i}\right)(p)\right)^{T}\right)$, $1 \leq i \leq m$; here $\left(A_{i} ; x\right)$ means that the $i-t h$ column of $A$ is substituted by the column vector $x$.

From (1), the solution of $(P C O D S)$ is given by

$Y(x)=\left[\mathcal{L}^{-1}\left(\frac{1}{\Delta(p)} \cdot \Delta_{i}(p)\right)\right]_{1 \leq i \leq m}^{T}$, a.e. $x \in I$.

Consider $\mathcal{L}(f)(p)=\left(\mathcal{L}\left(f_{i}\right)(p)\right)_{1 \leq i \leq m}^{T}$. Similarly, by $(P C)$, applying $\mathcal{L}$, it becomes

$$
p \cdot \mathcal{Y}(p)-Y(0)=A \cdot \mathcal{Y}(p)+\mathcal{L}(f)(p),
$$

in vectorial form $\left(p \cdot I_{m}-A\right) \cdot \mathcal{Y}(p)=\tilde{Y}_{0}+\mathcal{L}(f)(p)$, thus $\mathcal{Y}(p)=\left(p \cdot I_{m}-A\right)^{-1} \cdot\left[\tilde{Y}_{0}+\mathcal{L}(f)(p)\right]$, hence $Y(x)=\mathcal{L}^{-1}\left(\left(p \cdot I_{m}-A\right)^{-1}\right) \cdot \tilde{Y}_{0}+\mathcal{L}^{-1}\left(\left(p \cdot I_{m}-\right.\right.$ $\left.A)^{-1}\right) * f$.

By the power expansion on components, a particular case of Neumann's inverse theorem in finite dimensional space, one has $\left(p \cdot I_{m}-A\right)^{-1}$

$$
\begin{aligned}
= & (1 / p) \cdot\left[I_{m}-(1 / p) \cdot A\right]^{-1} \\
= & (1 / p) \cdot\left[I_{m}+(1 / p) \cdot A+((1 / p) \cdot A)^{2}\right. \\
& \left.\quad+\ldots+((1 / p) \cdot A)^{n}+\ldots\right] \\
= & \sum_{j=0}^{\infty} \frac{1}{p^{j+1}} \cdot A^{j},
\end{aligned}
$$


for $(1 / p) \cdot\|A\|_{M_{n}(\mathbb{R})}<1$. Hence, $\mathcal{L}^{-1}\left(\left(p \cdot I_{m}-A\right)^{-1}\right)$

$$
\begin{aligned}
& =\mathcal{L}^{-1}\left(\sum_{j=0}^{\infty} \frac{1}{p^{j+1}} \cdot A^{j}\right)=\sum_{j=0}^{\infty} A^{j} \cdot \mathcal{L}^{-1}\left(\frac{1}{p^{j+1}}\right) \\
& =\sum_{j=0}^{\infty} A^{j} \cdot \frac{1}{j !} x^{j}=\sum_{j=0}^{\infty} \frac{1}{j !} \cdot(A \cdot x)^{j}=\exp (A \cdot x) .
\end{aligned}
$$

Therefore,

$$
Y(x)=\exp (A \cdot x) \cdot \tilde{Y}_{0}+\exp (A \cdot x) * f,
$$

where in the convolution operation, Riemann integral(s) addmitts/admit $f$ piecewise continuous, not just $C^{0}\left(I, \mathbb{R}^{m}\right)$ (see [9], pg. 49).

II. By Lagrange's method, since its solution is of the form $Y=Y_{0}+Y_{p}$, with $Y_{0}(x)=\exp (A \cdot x) \cdot C$, $C=\left(C_{1}, \ldots, C_{m}\right)^{T} \in \mathbb{R}^{m}$, where $\exp (A \cdot x)=I_{m}+$ $\sum_{j=1}^{\infty} A^{j} \cdot x^{j} \cdot \frac{1}{j !}$, then $Y_{p}(x)=\exp (A \cdot x) \cdot C(x)$ thus $C^{\prime}(x)=\exp (-A \cdot x) \cdot f$, hence $C(x)=\int_{0}^{x} \exp (-A$. $s) \cdot f(s) d s$, so

$$
\begin{aligned}
Y_{p}(x) & =\exp (A \cdot x) \cdot \int_{0}^{x} \exp (-A \cdot s) \cdot f(s) d s \\
& =\int_{0}^{x} \exp [A \cdot(x-s)] \cdot f(s) d s \\
& =\exp (A \cdot x) * f .
\end{aligned}
$$

By the initial condition one obtains (3).

III. Suppose that the discontinuity points of $f_{i}$ are jump points, that is $f_{i}\left(x_{k}-\right), f_{i}\left(x_{k}+\right)$ are real not equal, for any fixed $i \in\{1, \ldots, m\}$ and $k \in N$. By the successive approach, since $f \in C^{0}\left(I_{0}, \mathbb{R}^{m}\right)$ one has (classic) solution on $I_{0}$ given by

$$
\begin{aligned}
(3)_{0} & \bar{Y}_{0}(x)=\exp (A \cdot x) \cdot \tilde{Y}_{0} \\
+ & \left.\int_{0}^{x} \exp [A \cdot(x-s)] \cdot f\right|_{I_{0}}(s) d s, \forall x \in I_{0} .
\end{aligned}
$$

Take $\bar{Y}\left(x_{1}\right)=\lim _{x} x_{1} \bar{Y}_{0}(x)$ and consider the Cauchy problem on $I_{1}$

$$
(P C)_{1}\left\{\begin{array}{l}
Y^{\prime}=A \cdot Y+\left.f\right|_{I_{1}} \\
Y\left(x_{1}\right)=\bar{Y}\left(x_{1}\right)
\end{array}\right.
$$

with solution

$$
\begin{aligned}
(3)_{1} \quad & \bar{Y}_{1}(x)=\exp (A \cdot x) \cdot \bar{Y}\left(x_{1}\right) \\
+ & \left.\int_{x_{1}}^{x} \exp [A \cdot(x-s)] \cdot f\right|_{I_{1}}(s) d s, \forall x \in I_{1} .
\end{aligned}
$$

Taking into account the solutions on $I_{0}$ and $I_{1}$, respectively, $(3)_{0}$ and $(3)_{1}$, by mathematical induction, suppose that for $1<k_{0}<\operatorname{card} N$,

$$
\begin{aligned}
(3)_{k_{0}} & \bar{Y}_{k_{0}}(x)=\exp (A \cdot x) \cdot \bar{Y}\left(x_{k_{0}}\right) \\
& +\left.\int_{x_{k_{0}}}^{x} \exp [A \cdot(x-s)] \cdot f\right|_{I_{k_{0}}}(s) d s,
\end{aligned}
$$

$\forall x \in I_{k_{0}}$, is the solution on $I_{k_{0}}$. Take $\bar{Y}\left(x_{k_{0}+1}\right)=$ $\lim _{x \nearrow x_{k_{0}+1}} \bar{Y}_{k_{0}}(x)$ and consider the Cauchy problem on $I_{k_{0}+1}$

$$
(P C)_{k_{0}+1}\left\{\begin{array}{l}
Y^{\prime}=A \cdot Y+\left.f\right|_{I_{k_{0}+1}} \\
Y\left(x_{k_{0}+1}\right)=\bar{Y}\left(x_{k_{0}+1}\right)
\end{array}\right.
$$

with solution

$$
\begin{aligned}
(3)_{k_{0}+1} & \bar{Y}_{k_{0}+1}(x)=\exp (A \cdot x) \cdot \bar{Y}\left(x_{k_{0}+1}\right) \\
+ & \left.\int_{x_{k_{0}}+1}^{x} \exp [A \cdot(x-s)] \cdot f\right|_{I_{k_{0}+1}}(s) d s
\end{aligned}
$$

$\forall x \in I_{k_{0}+1}$.

Consequently, from $(3)_{k_{0}}$ and $(3)_{k_{0}+1}$, the solution of $(P C O D S)$ is given by

$$
\bar{Y}(x)=\left\{\begin{array}{l}
\bar{Y}_{0}(x), \quad x \in I_{0} \\
\bar{Y}_{1}(x), \quad x \in I_{1} \\
\ldots \\
\bar{Y}_{k_{0}}(x), \quad x \in I_{k_{0}} \\
\bar{Y}_{k_{0}+1}(x), \quad x \in I_{k_{0}+1} \\
\ldots
\end{array}\right.
$$

\section{Examples}

Let $m=1, A=1$, and $f(x)=H(1-x)$, where $H$ is the Heaviside function. By basic calculus, the solution is given by

$$
\begin{aligned}
y(x) & =\exp (x)+\exp (x) * H(1-x) \\
& =\exp (x)+\int_{0}^{x} \exp (x-s) \cdot H(1-s) d s \\
& = \begin{cases}2 \cdot e^{x}-1, & x<1 \\
\left(2-e^{-1}\right) \cdot e^{x}, & x \geq 1 .\end{cases}
\end{aligned}
$$

The successive approach was expressed in [2], in order to obtain the same solution.

For $f(x)=H(x)$, the solution is given by

$$
\begin{aligned}
y(x) & =\exp (x)+\exp (x) * H(x) \\
& = \begin{cases}\exp (x), & x<0 \\
2 \cdot \exp (x)-1, & x \geq 0 .\end{cases}
\end{aligned}
$$

Let $m=2, x_{1}>0, A=\left(\begin{array}{ll}a_{11} & a_{12} \\ a_{21} & a_{22}\end{array}\right)=$ $\left(\begin{array}{cc}0 & 1 \\ -1 & 0\end{array}\right), f_{1}=\chi_{\left(x_{1},+\infty\right)}=H\left(\cdot-x_{1}\right), f_{2}=2 f_{1}$, and the Cauchy problem

$$
(*)\left\{\begin{array}{l}
y_{1}^{\prime}=a_{11} \cdot y_{1}+a_{12} \cdot y_{2}+f_{1} \\
y_{2}^{\prime}=a_{21} \cdot y_{1}+a_{22} \cdot y_{2}+f_{2} \\
y_{1}(0)=\tilde{y}_{1}=0, y_{2}(0)=\tilde{y}_{2}=1 .
\end{array}\right.
$$


By using the three methods, one by one, the following are to be obtained. By (2) its solution is $Y(x)=\left[y_{1}(x), y_{2}(x)\right]^{T}$ given by

$$
\begin{aligned}
& y_{1}(x)=\mathcal{L}^{-1}\left(\frac{1}{\Delta(p)} \cdot \Delta_{1}(p)\right), \\
& y_{2}(x)=\mathcal{L}^{-1}\left(\frac{1}{\Delta(p)} \cdot \Delta_{2}(p)\right),
\end{aligned}
$$

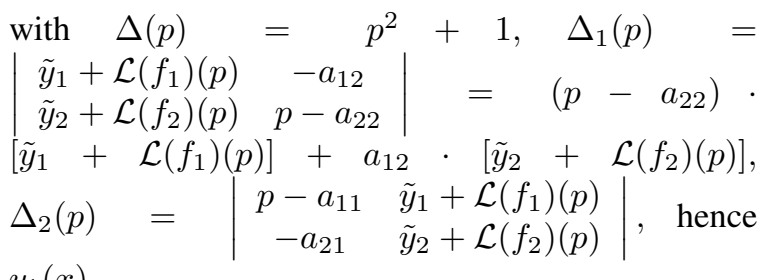
$y_{1}(x)$

$$
\begin{aligned}
=\quad \tilde{y}_{1} & \cdot \mathcal{L}^{-1}\left[\frac{1}{\Delta(p)} \cdot\left(p-a_{22}\right)\right] \\
& +\mathcal{L}^{-1}\left[\frac{1}{\Delta(p)} \cdot\left(p-a_{22}\right)\right] * f_{1} \\
& +a_{12} \cdot \tilde{y}_{2} \cdot \mathcal{L}^{-1}\left(\frac{1}{\Delta(p)}\right) \\
& +a_{12} \cdot \mathcal{L}^{-1}\left(\frac{1}{\Delta(p)}\right) * f_{2} \\
=\quad & \cos x * f_{1}+\sin x+\sin x * 2 f_{1} \\
=\quad & \sin x+\int_{0}^{x} \cos (x-s) \cdot f_{1}(s) d s \\
& +2 \int_{0}^{x} \sin (x-s) \cdot f_{1}(s) d s \\
= & \left\{\begin{array}{l}
\sin x, x<x_{1} \\
\sin x+\sin \left(x-x_{1}\right)-2 \cos \left(x-x_{1}\right)+2, \\
x>x_{1}
\end{array}\right.
\end{aligned}
$$

and $y_{2}(x)$

$$
\begin{aligned}
&=a_{21} \cdot \tilde{y}_{1} \cdot \mathcal{L}^{-1}\left(\frac{1}{\Delta(p)}\right) \\
& \quad+\tilde{y}_{2} \cdot \mathcal{L}^{-1}\left[\frac{1}{\Delta(p)} \cdot\left(p-a_{11}\right)\right] \\
&+a_{21} \cdot \mathcal{L}^{-1}\left(\frac{1}{\Delta(p)}\right) * f_{1} \\
&+\mathcal{L}^{-1}\left[\frac{1}{\Delta(p)} \cdot\left(p-a_{11}\right)\right] * f_{2} \\
&=\quad \cos x-\sin x * f_{1}+\cos x * 2 f_{1} \\
&=\quad \cos x-\int_{0}^{x} \sin (x-s) \cdot f_{1}(s) d s \\
&+2 \int_{0}^{x} \cos (x-s) \cdot f_{1}(s) d s \\
&=\left\{\begin{array}{l}
\cos x, x<x_{1} \\
\cos x+\cos \left(x-x_{1}\right)-1+2 \sin \left(x-x_{1}\right), \\
x>x_{1} .
\end{array}\right.
\end{aligned}
$$

Using the Lagrange's method, the same solution is going to be obtained. Since $\exp (A \cdot x)=I_{2}+$ $\sum_{k=1}^{\infty} \frac{1}{k !} \cdot A^{k} \cdot x^{k}=\left(\begin{array}{cc}\cos x & \sin x \\ -\sin x & \cos x\end{array}\right)$, by (3) the solution is $Y(x)$

$$
\begin{aligned}
& =\exp (A \cdot x) \cdot \tilde{Y}_{0}+\exp (A \cdot x) * f \\
& =\left(\begin{array}{cc}
\cos x & \sin x \\
-\sin x & \cos x
\end{array}\right) \cdot\left(\begin{array}{l}
0 \\
1
\end{array}\right) \\
& +\left(\begin{array}{cc}
\cos x & \sin x \\
-\sin x & \cos x
\end{array}\right) *\left(f_{1}, f_{2}\right)^{T} \\
& =\left(\begin{array}{c}
\sin x \\
\cos x
\end{array}\right) \\
& +\int_{0}^{x}\left(\begin{array}{cc}
\cos (x-s) & \sin (x-s) \\
-\sin (x-s) & \cos (x-s)
\end{array}\right) \cdot\left(\begin{array}{l}
f_{1}(s) \\
f_{2}(s)
\end{array}\right) d s \\
& =\left(\begin{array}{c}
\sin x \\
\cos x
\end{array}\right)
\end{aligned}
$$

$+\left(\begin{array}{c}\int_{0}^{x}\left[\cos (x-s) \cdot f_{1}(s)+\sin (x-s) \cdot f_{2}(s)\right] d s \\ \int_{0}^{x}\left[-\sin (x-s) \cdot f_{1}(s)+\cos (x-s) \cdot f_{2}(s)\right] d s\end{array}\right)$

$$
\begin{aligned}
& =\left(\begin{array}{c}
\sin x \\
\cos x
\end{array}\right) \\
& +\left(\begin{array}{c}
\int_{0}^{x} \cos (x-s) \cdot H\left(s-x_{1}\right) d s \\
-\int_{0}^{x} \sin (x-s) \cdot H\left(s-x_{1}\right) d s
\end{array}\right) \\
& +\left(\begin{array}{l}
2 \int_{0}^{x} \sin (x-s) \cdot H\left(s-x_{1}\right) d s \\
2 \int_{0}^{x} \cos (x-s) \cdot H\left(s-x_{1}\right) d s
\end{array}\right) \\
& =\left(\begin{array}{l}
\sin x \\
\cos x
\end{array}\right) \\
& +\left(\begin{array}{c}
\int_{x_{1}}^{x} \cos (x-s) d s+2 \int_{x_{1}}^{x} \sin (x-s) d s \\
-\int_{x_{1}}^{x} \sin (x-s) d s+2 \int_{x_{1}}^{x} \cos (x-s) d s
\end{array}\right) \\
& =\left\{\begin{array}{l}
\left(\begin{array}{c}
\sin x \\
\cos x
\end{array}\right), \\
x<x_{1} \\
\left(\begin{array}{l}
\sin x+\sin \left(x-x_{1}\right)-2 \cos \left(x-x_{1}\right)+2 \\
\cos x+\cos \left(x-x_{1}\right)-1+2 \sin \left(x-x_{1}\right)
\end{array}\right), \\
x>x_{1}
\end{array}\right.
\end{aligned}
$$

On the other hand, by successive approach, on $I_{0}=$ $\left[0, x_{1}\right),(P C)_{I_{0}}$ is

$$
\left\{\begin{array}{l}
y_{1}^{\prime}(x)=a_{11} \cdot y_{1}(x)+a_{12} \cdot y_{2}(x)=y_{2}(x) \\
y_{2}^{\prime}(x)=a_{21} \cdot y_{1}(x)+a_{22} \cdot y_{2}(x)=-y_{1}(x) \\
y_{1}(0)=0, y_{2}(0)=1,
\end{array}\right.
$$

the solution is $\left(\bar{y}_{1}(x), \bar{y}_{2}(x)\right)=\bar{Y}_{0}(x)=$ $(\sin x, \cos x)$. The Cauchy problem $(P C)_{I_{1}}$ on $I_{1}=$ $\left[x_{1},+\infty\right)$ is

$$
\begin{cases}Y^{\prime} & =A \cdot Y+\left.f\right|_{I_{1}} \\ Y\left(x_{1}\right) & =\bar{Y}_{0}\left(x_{1}-\right)=\bar{Y}\left(x_{1}\right) \\ & =\lim _{x \nearrow x_{1}} \bar{Y}_{0}(x)=\left(\sin x_{1}, \cos x_{1}\right),\end{cases}
$$

with solution $\bar{Y}_{1}(x)$

$=\left(\begin{array}{c}-2 \cdot \cos \left(x-x_{1}\right)+\sin \left(x-x_{1}\right)+\sin x+2 \\ 2 \cdot \sin \left(x-x_{1}\right)+\cos \left(x-x_{1}\right)+\cos x-1\end{array}\right)$. 
The solution of $(*)$, expressed in (4), is

$$
\bar{Y}(x)= \begin{cases}\bar{Y}_{0}(x), & x \in I_{0} \\ \bar{Y}_{1}(x), & x \in I_{1}\end{cases}
$$

As it was mentioned before, a substitution $y_{2}=$ $y_{1}^{\prime}-f_{1}$, except the angular point $x_{1}$, leads to

$$
y_{1}^{\prime \prime}-0=-y_{1}+f_{2} \text {, a.e. on }[0,+\infty),
$$

that is $y_{1}^{\prime \prime}+y_{1}=f_{2}$, so

$y_{1}(x)= \begin{cases}C_{1} \cdot \cos x+C_{2} \cdot \sin x, & x \in\left[0, x_{1}\right) \\ \tilde{C}_{1} \cdot \cos x+\tilde{C}_{2} \cdot \sin x+2, & x \in\left(x_{1},+\infty\right),\end{cases}$

therefore $y_{2}(x)=y_{1}^{\prime}(x)-f_{1}(x)=$ $\begin{cases}-C_{1} \cdot \sin x+C_{2} \cdot \cos x, & x \in\left[0, x_{1}\right) \\ -\tilde{C}_{1} \cdot \sin x+\tilde{C}_{2} \cdot \cos x-1, & x \in\left(x_{1},+\infty\right) .\end{cases}$

By the initial conditions $y_{1}(0)=C_{1}=0, y_{2}(0)=$ $C_{2}=1$, the solution is $\bar{Y}_{0}(x)=(\sin x, \cos x)$ on $I_{0}$. To preserve continuity of $\bar{Y}=\left(\bar{y}_{1}, \bar{y}_{2}\right)^{T}$ one has $\begin{cases}\tilde{C}_{1} \cdot \cos x+\tilde{C}_{2} \cdot \sin x+2 & =\sin x_{1} \\ -\tilde{C}_{1} \cdot \sin x_{1}+\tilde{C}_{2} \cdot \cos x_{1}-1 & =\cos x_{1}\end{cases}$ so $\tilde{C}_{1}=-2 \cdot \cos x_{1}-\sin x_{1}, \tilde{C}_{2}=-2 \cdot \sin x_{1}+$ $\cos x_{1}+1$, therefore $\bar{y}_{1}(x)$

$= \begin{cases}\sin x, & x \in\left[0, x_{1}\right) \\ -2 \cdot \cos \left(x-x_{1}\right) & \\ +\sin \left(x-x_{1}\right)+\sin x+2, & x \in\left(x_{1},+\infty\right),\end{cases}$

and $\bar{y}_{2}(x)$

$= \begin{cases}\cos x, & x \in\left[0, x_{1}\right) \\ 2 \cdot \sin \left(x-x_{1}\right) & \\ \quad+\cos \left(x-x_{1}\right)+\cos x-1, & x \in\left(x_{1},+\infty\right) .\end{cases}$

Observe that, if one considers incorrectly as solution $y_{1}(x)$ = $\left\{\begin{array}{l}C_{1} \cdot \cos x+C_{2} \cdot \sin x, \quad x \in\left[0, x_{1}\right) \\ C_{1} \cdot \cos x+C_{2} \cdot \sin x+2,\end{array}\right.$ $C_{1} \cdot \cos x+C_{2} \cdot \sin x+2, \quad x \in\left(x_{1},+\infty\right)$ (from $y_{1}^{\prime \prime}+y_{1}=f_{2}$ ), by initial conditions it obtains $y_{1}^{*}(x)=\left\{\begin{array}{ll}\sin x, & x \in\left[0, x_{1}\right) \\ \sin x+2, & x \in\left(x_{1},+\infty\right)\end{array}\right.$ and $y_{2}^{*}(x)=\left\{\begin{array}{ll}\cos x, & x \in\left[0, x_{1}\right) \\ \cos x-1, & x \in\left(x_{1},+\infty\right) .\end{array}\right.$ Although $\left(y_{1}^{*}, y_{2}^{*}\right)$ verifies $(*)$ on $\left[0, x_{1}\right)$ and $\left(x_{1},+\infty\right)$, respectively, it is not solution according to Definition 1, because $\left(y_{1}^{*}, y_{2}^{*}\right) \notin C\left(I, \mathbb{R}^{2}\right)$.

\section{The solution returned by a computer algebra system}

Utilising the computer algebra system WolframAlpha [15], the commands to obtain solution in the Examples section, are entered.
For $m=1$, by description $y=y(x)$, the equation is the following:

$$
y^{\prime}(x)=y(x)+H e a v i s i d e(x), y(0)=1
$$

The displayed solution is

$$
\left(e^{x}-1\right) \cdot \theta(x)+e^{x},
$$

that coincide with the one given in (5), where $\theta(\cdot)=$ Heaviside $(\cdot)$

For $m=2, y_{1}=y_{1}(x), y_{2}=y_{2}(x)$, the commands are the following:

$y 1^{\prime}(x)=y 2(x), y 2^{\prime}(x)=-y 1(x), y 1(0)=0, y 2(0)=1$

The displayed solution on $I_{0}$ is

$$
y 1(x)=\sin x, y 2(x)=\cos x .
$$

Compute the limits at $x_{1}-, \quad \lim _{x} \nearrow x_{1} y_{1}(x)$, $\lim _{x \nearrow x_{1}} y_{2}(x)$ by the command

$\lim \sin x$ as $x_{->x 1-,}$ im $\cos x$ as $x->x 1-$

The displayed answer is

$$
\{\sin x 1, \cos x 1\}
$$

The commands on $I_{1}$ are the following:

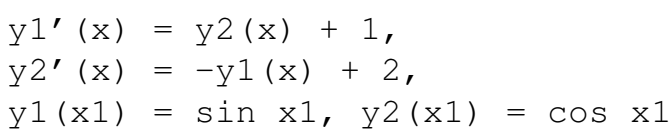

At this stage WA encounters some difficulties to display a solution that is not the case by writing it. Anyway, it is given in (6).

The solution can be obtained directly by the commands (in one line)

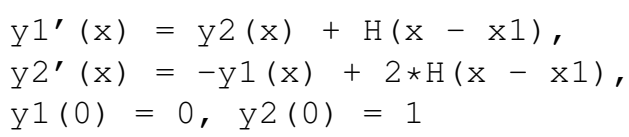

Here, the input contains ' $*$ ' instead of '. , for multiplication, that should not be confused with the convolution operation. 
The displayed solution is

$$
\left\{\begin{array}{rl}
y & 1(x)=2 \theta(x-x 1)-2 \sin (x) \sin (x 1) \theta(x-x 1) \\
& +\theta(-x 1) \sin (x)+2 \theta(-x 1) \sin (x) \sin (x 1) \\
& -2 \cos (x) \cos (x 1) \theta(x-x 1)-2 \theta(-x 1) \cos (x) \\
& +2 \theta(-x 1) \cos (x) \cos (x 1) \\
& +\sin (x) \cos (x 1) \theta(x-x 1) \\
& -\cos (x) \sin (x 1) \theta(x-x 1) \\
& -\theta(-x 1) \sin (x) \cos (x 1) \\
& +\theta(-x 1) \cos (x) \sin (x 1)+\sin (x) \\
y & 2(x)=-\theta(x-x 1)+2 \theta(-x 1) \sin (x) \\
& +\sin (x) \sin (x 1) \theta(x-x 1) \\
& -\theta(-x 1) \sin (x) \sin (x 1) \\
& +\cos (x) \cos (x 1) \theta(x-x 1) \\
& -\theta(-x 1) \cos (x) \cos (x 1) \\
& +\theta(-x 1) \cos (x)-2 \cos (x) \sin (x 1) \theta(x-x 1) \\
& +2 \theta(-x 1) \cos (x) \sin (x 1) \\
& +2 \sin (x) \cos (x 1) \theta(x-x 1) \\
& -2 \theta(-x 1) \sin (x) \cos (x 1)+\cos (x),
\end{array}\right.
$$

that is, and since $-x 1<0$, it should be read as

$$
\left\{\begin{array}{c}
y_{1}(x)=\sin (x)+2 \cdot \theta(x-x 1) \\
-2 \cdot \theta(x-x 1) \cdot \cos (x-x 1) \\
+\theta(x-x 1) \cdot \sin (x-x 1) \\
y_{2}(x)=\cos (x)-\theta(x-x 1) \\
+\cos (x-x 1) \cdot \theta(x-x 1) \\
+2 \sin (x-x 1) \cdot \theta(x-x 1)
\end{array}\right.
$$

that coincide with the one given in (6).

\section{Distributional solutions}

In this section we aim to follow how the discontinuities can be absorbed alternatively. Let us consider the example from Section 3.

Let $\left(f_{1 n}\right)_{n \in \mathbb{N}}, \quad\left(f_{2 n}\right)_{n \in \mathbb{N}}$ be two sequences of continuous functions, $f_{1 n}, f_{2 n}: I \rightarrow \mathbb{R}$, such that $f_{1 n} \stackrel{n \rightarrow \infty}{\longrightarrow} f_{1}$ (pointwise), and $f_{2 n} \stackrel{n \rightarrow \infty}{\longrightarrow} f_{2}$, respectively. For example, $f_{1 n}(x)=$ $\begin{cases}0, & x \in\left[0, x_{1}-\frac{1}{n}\right)=\left[0, \alpha_{n}\right) \\ n x-n x_{1}+1, & x \in\left[x_{1}-\frac{1}{n}, x_{1}\right) \\ 1, & x \in\left[x_{1},+\infty\right) ;\end{cases}$ $f_{2 n}=2 \cdot f_{1 n}$. For each $n \in \mathbb{N}$, let us consider

$$
(P C O D S)_{n}\left\{\begin{array}{l}
y_{1}^{\prime}=a_{11} \cdot y_{1}+a_{12} \cdot y_{2}+f_{1 n} \\
y_{2}^{\prime}=a_{21} \cdot y_{1}+a_{22} \cdot y_{2}+f_{2 n} \\
y_{1}(0)=0, y_{2}(0)=1
\end{array}\right.
$$

having the classic solution $\left(y_{1 n}, y_{2 n}\right)$ given by

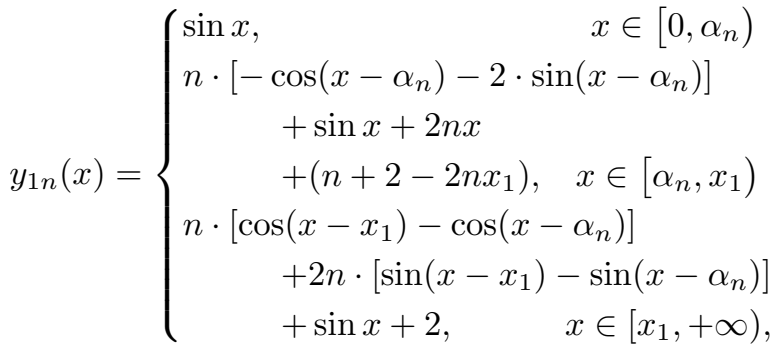

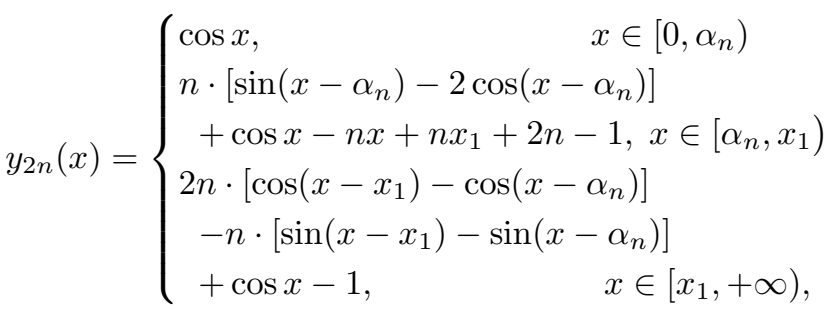

and $\left(y_{1 n}, y_{2 n}\right) \stackrel{n \rightarrow \infty}{\longrightarrow}\left(y_{1}, y_{2}\right)$, the solution given in (6). On the other hand $y_{1 n} \not y_{1}$ (uniformly).

The way to the space of distribution is known (see $[7,13])$. Firstly, it is defined

$$
\Sigma(\mathbb{R})=\{y: \mathbb{R} \rightarrow \mathbb{R} \mid y \text { measurable a.e. on } \mathbb{R}\},
$$

relation $\sigma$ on $\Sigma$ given by $y \sigma z \Longleftrightarrow y(x)=z(x)$ a.e. on $\mathbb{R}$;

$$
S(\mathbb{R})=\Sigma(\mathbb{R}) / \sigma,
$$

$L_{l o c}^{1}(\mathbb{R})=\left\{y \in S(\mathbb{R})\left|\int_{C}\right| y(x) \mid d x<+\infty, \forall C \subset\right.$ $\mathbb{R}$ compact $\}$, the fundamental space

$$
\mathcal{D}=\{y: \mathbb{R} \rightarrow \mathbb{R} \mid \operatorname{supp}(y) \text { compact }\} \cap C^{\infty}(\mathbb{R}),
$$

and its dual

$$
\mathcal{D}^{*}=\left\{y^{*}: \mathcal{D} \rightarrow \mathbb{R} \mid y^{*} \text { linear and continuous }\right\} .
$$

The convergence of $\left(y_{n}^{*}\right)_{n \in \mathbb{N}}$ to $y^{*}$ in $\mathcal{D}^{*}$ is given by $\frac{d^{j}}{d x^{j}} y_{n}^{*}(u) \rightarrow \frac{d^{j}}{d x^{j}} y^{*}(u),($ in $\mathbb{R}) \forall u \in \mathcal{D}, \forall j \in \mathbb{N} \cup\{0\}$.

Let $\left(y_{1 n}, y_{2 n}\right)$ be the classic solution for $(\operatorname{PCODS})_{n}$ :

$$
\left\{\begin{array}{l}
y_{1 n}^{\prime}=y_{2 n}+f_{1 n} \\
y_{2 n}^{\prime}=-y_{1 n}+f_{2 n} \\
y_{1}(0)=0, y_{2}(0)=1 .
\end{array}\right.
$$

By the identification, $g=y_{g}^{*}$, for $g$ continuous on $\mathbb{R} \backslash N$, thus $g \in L_{l o c}^{1}(\mathbb{R})$, one can write

$$
(O D S)_{n}^{*}\left\{\begin{array}{l}
y_{\frac{d}{d x} y_{1 n}}^{*}=y_{y_{2 n}}^{*}+y_{f_{1 n}}^{*} \\
y_{\frac{d}{d x} y_{2 n}}^{*}=-y_{y_{1 n}}^{*}+y_{f_{2 n}}^{*} .
\end{array}\right.
$$

For arbitrary $u \in \mathcal{D}$ one has $\frac{d}{d x} y_{y}^{*}(u)=y_{\frac{d}{d x} y}^{*}(u)$, so $(O D S)_{n}^{*}$ becomes

$$
\left\{\begin{array}{l}
\frac{d}{d x} y_{y_{1 n}}^{*}(u)=y_{y_{2 n}}^{*}(u)+y_{f_{1 n}}^{*}(u) \\
\frac{d}{d x} y_{y_{2 n}}^{*}(u)=-y_{y_{1 n}}^{*}(u)+y_{f_{2 n}}^{*}(u) .
\end{array}\right.
$$


Since $y_{y_{1 n}}^{*} \stackrel{n \rightarrow \infty}{\longrightarrow} y_{y_{1}}^{*}, y_{y_{2 n}}^{*} \stackrel{n \rightarrow \infty}{\longrightarrow} y_{y_{2}}^{*}$ (pointwise), by taking $n \rightarrow \infty$, it obtains in $\mathcal{D}^{*}$

$$
\left\{\begin{array}{l}
\frac{d}{d x} y_{y_{1}}^{*}=y_{y_{2}}^{*}+y_{f_{1}}^{*} \\
\frac{d}{d x} y_{y_{2}}^{*}=-y_{y_{1}}^{*}+y_{f_{2}}^{*}
\end{array}\right.
$$

and by the same isomorphism it follows that

$$
\left\{\begin{array}{l}
y_{1}^{\prime}=y_{2}+f_{1} \\
y_{2}^{\prime}=-y_{1}+f_{2} \\
y_{1}(0)=0, y_{2}(0)=1 .
\end{array}\right.
$$

Note that if the function $f$ is only Lebesgue integrable and not Rienmann integrable, for example $f=1_{\mathbb{Q}_{+}}$that has the set of discontinuities $[0,+\infty)$, the successive approach is not applicable. More, this function has no jump points. Distributional solution is therefore to be obtained if $f$ is the free term.

\section{Conclusions}

The notion of classic solution for a Cauchy problem given by a nonhomogeneous linear differential system is extended. The nonhomogeneity is given by a free term considered to be continuous except a most countable set of jump points. The solution can be obtained by the present new method that consists on solving successive Cauchy problems governed by the differential system with continuous free term.

The Laplace transform was used to solve differential equations that involves fractional derivatives (see [6]). The idea of sequentiality can be extended for Cauchy problems with nonhomogeneous differential equations $([5,12]$ can be consulted and the reference therein), in particular for $\left\{\begin{array}{l}{ }^{C} D^{\alpha} y=f \\ y(0)=y_{0},\end{array}\right.$ where $f$ has some immediate integrable expression(s) on the intervals where it is continuous.

The computer algebra system WolframAlpha was used. A similar approach can be developed by using Python (Anaconda2, Jupyter Notebook, [14]).

\section{References}

[1] Bogdan, M. (2018), An exemplified introduction of the Laplace transform, Scientiffic Bulletin of the "Petru Maior" University, 15(1), pp. 25-28.

[2] Bogdan, M. Ispas, I. (2019), An implementation attempt for solving Cauchy problem with a sequential approach, Procedia Manufacturing 32, pp. 634-639.

[3] Haimovici, A. (1965), Ecuații diferenţiale şi ecuaţii integrale, Editura Didactică şi Pedagogică, Bucureşti (in Romanian).
[4] Kanwal, R.P. (2004), Generalized Equations: Theory and Applications, 3rd edition, Springer.

[5] Ishteva, M.K. (2005), Properties and applications of the Caputo fractional operator, (Master thesis) http : //homepages.vub.ac.be/ mishteva/papers/ Ishteva_MScThesis.pdf (2020).

[6] Jarad, F., Abdeljawad, T. (2020), Generalized fractional derivatives ans Laplace transform, Descrete and Continuous Dynamical Systems 13(3), pp. 709-722.

[7] Muntean, I. (1993), Analiză funcţională, preprint, Cluj-Napoca (in Romanian).

[8] Olariu, V., Prepeliţă, V. (1985), Matematici speciale, Editura didactică şi pedagogică, Bucureşti (in Romanian).

[9] Olariu, V., Stănăşilă, T. (1982), Ecuații diferenţiale şi cu derivate parţiale, Editura tehnică, Bucureşti (in Romanian).

[10] Pavel, G., Tomuţa, F.I., Gavrea, I. (1981), Matematici speciale, Editura Dacia, ClujNapoca (in Romanian).

[11] Redheffer, R. (1991) Differential Equations: Theory and Applications, Boston.

[12] Sakar, M.G., Saldir, O., Akgül, A. (2018), Numerical solutions of fractional Bratu type equations with Legendre reproducing kernel method, Int. J. Appl. Comput. Math. 4(126), pp. 1-14.

[13] Vladimirov, V.S. (1981), Culegere de probleme de ecuaţiile fizicii matematice, Editura ştiinţifică şi enciclopedică, Bucureşti (in Romanian).

[14] Python, https://www.python.org/ (2019)

[15] WolframAlpha, https://www.wolframalpha.com/, (2020) 